\title{
FINAL REPORT FOR DE-FG02-03ER46071 ENTITLED, "UNDERSTANDING FOAM RHEOLOGY FROM THE MICROSCOPIC TO THE MACROSCOPIC SCALE"
}

\section{Brief Summary of Research Goals}

This research effort is focused on understanding the mechanical response of foams, and other complex fluids, from the microscopic to the macroscopic level. The research uses a model two-dimensional system: bubble rafts. Bubble rafts are a single layer of gas bubbles with liquid walls that float on a water surface. The work involves studies of the macroscopic response of foam under various conditions of external forcing, mesoscopic studies of bubble motion, and systematic variations of the microscopic details of the system.

In addition to characterizing the specific properties of the bubble raft, a second aim of the research is to provide experimental tests of various general theories that have recently been developed to characterize complex fluids. Primarily, the focus is on testing the proposed jamming phase diagram paradigm. This paradigm suggests that a general "jammed" state of matter exists and is common to a wide range of systems, including foam, colloids, granular matter, glasses, and emulsions. Therefore, as discussed in the previous report, we have extended our research in two directions. First, we have included studies of plastic bead rafts. These are systems of plastic beads floating on the air-water interface. The advantage of plastic beads is that they do not pop, so they can be studied for the much longer periods of time required to measure the slow dynamics associated with the jammed state. Also, they allow us to explore a different density regime than the bubbles. Second, to better understand the role of defects in jamming behavior, we have done a few experiments on the impact of defects on domain growth. This will be discussed in more detail in the later sections.

\section{Research Accomplishments}

\section{A. Summary of papers and presentations}

In the last report, we did not have the complete reference for one paper:

Statistics of Bubble Rearrangements in a Slowly Sheared Two-dimensional Foam, M. Dennin, Phys. Rev. E 70, 041406 (2004).

There were three papers published during the previous year:

Velocity Fluctuations in a Slowly Sheared Bubble Raft, M. Dennin, Colloids and Surfaces, A: Physiochem. And Eng. Asp. 263, 76 (2005).

Slow Steady Shear of Plastic Bead Rafts, Michael Twardos and Michael Dennin, Granular Matter 7, 92 (2005).

Comparison between step strains and slow steady shear in a bubble raft, M. Twardos and M. Dennin, Phys. Rev. E 71, 061402 (2005). 
Impact of noise on domain growth in electroconvection, M. Griffith and M. Dennin, Phys. Rev. E 74, 027201 (2006).

Impact of boundaries on velocity profiles in bubble rafts, Y. Wang, K. Krishan, and M. Dennin, Phys. Rev. E 73, 031401 (2006).

Asymmetric response of a jammed plastic bead raft Michael Twardos, Michael Dennin, Phys. Rev. Lett. 97, 110601 (2006).

Bubble kinematics in a sheared foam, Yuhong Wang, Kapilanjan Krishan, Michael Dennin, Phys. Rev. E 74, 041405 (2006).

Flow transitions in two-dimensional foams, Christopher Gilbreth, Scott Sullivan, Michael Dennin, Phys. Rev. E 74, 051406 (2006).

Statistics of microscopic yielding in sheared aqueous foams, Yuhong Wang, Kapilanjan

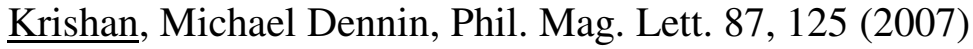

Limits of the Equivalence of Time and Ensemble Averages in Shear Flows, Y. Wang, K. Krishan, and M. Dennin, Phys. Rev. Lett. 98, 220602 (2007)

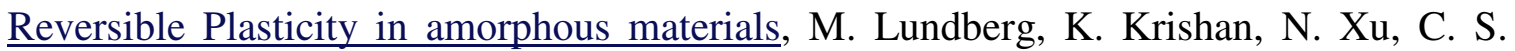
O’Hern, and M. Dennin, Phys. Rev. E 77, 041505 (2008).

One measure of the excitement of the work that has been accomplished is the number of invited talks over the previous year. I have been invited to give nine talks at various institutions and conferences. It is worth pointing out that this include three American Physical Society (APS) meetings (indicated by *) and one international conference (indicated by $* *)$. The international conference was a major meeting of the world's experts in two-dimensional foam rheology, and included a session that I chaired on charting the course of research in this area over the next 5 years.

Foam under stress: the response of foam to applied strain, UC Irvine Condensed Matter Seminar, UC Irvine, Nov. 10, 2004.

Foam under stress: the response of foam to applied strain, Department of Physics Colloquium, Cal. State Northridge, Dec. 1, 2004.

Is jammed matter a new phase: Experiments in flowing foam. Physical Chemistry Seminar, Department of Chemistry, UC Irvine, Jan 18, 2005.

Jamming and flow: bubble rafts under stress, Department of Physics Colloquium, University of Pittsburgh, Feb 7, 2005.

* Nonlinear Dynamics of Soft-Matter: Continuum Mechanics in the Classroom March Meeting of the American Physical Society, 2005. 
** Bubble motions in sheared bubble rafts: from irregular rearrangements to smooth flow, invited talk at the Foam Rheology in Two-Dimensions Meeting, Aberystwyth, Wales, June 27 - 28, 2005.

Continuum Mechanics: Research Questions for the Classroom, invited talk at the American Association of Physics Meeting, Utah, Aug. 2005.

* Foam: solid or liquid?, invited talk at the Ohio Section meeting of the American Physical Society, Oct. 2005.

* Flowing Foam: T1 events and solid-liquid transitions*, invited talk at the American Physical Society meeting of the Division of Fluid Dynamics, Nov. 2005.

In addition to the invited talks, there were two contributed presentations at the March Meeting of the American Physical Society.

Dynamics of Dislocations in Anisotropic Pattern, Carina Kamaga and Michael Dennin, presented at the March Meeting of the American Physical Society, 2005.

Step Strains in a Disordered Foam, Michael Twardos and Michael Dennin, presented at the March Meeting of the American Physical Society, 2005

B. Summary of major accomplishments

\section{i. Construction of new flow geometries}

As discussed in the annual reports, a critical goal of this project was the development of a set up for applying parallel shear to the bubble raft. Recall that our initial apparatus is a Couette trough, i.e. two concentric cylinders with the bubbles sheared between them. In a Couette geometry, the average stress varies as a function of the radial position. Therefore, the use of a parallel trough, in which the average stress is uniform, is critical for understanding the impact separating effects due to the spatial variation of stress and intrinsic effects.

We have accomplished the design and construction of such a trough. It uses two bands that can drive the bubbles, and has the flexibility to generate three different types of flow: driving walls moving in opposite directions, driving walls moving in the same direction at different speeds, and a single wall moving with the other wall held fixed. This flexibility allows us to study conditions of different symmetry in the system. Most of the work will be done in a configuration with walls moving in the opposite direction as this allows for study of continuous shear with no NET flow. The trough was also designed to allow for the placement of a top glass plate in contact with the bubbles. This was important for exploring the impact of the boundary conditions on the bubbles. This project is completed and is discussed in more detail below. 
In addition, we were able to construct a system for studying oscillatory shear using a modification of the parallel shear device. In this system, we have a single fixed wall and we oscillate a parallel wall. This has allowed us to explore a range of interesting dynamics as a function of amplitude and frequency. Our initial results in this area were published in Reversible Plasticity in amorphous materials, M. Lundberg, K. Krishan, N. Xu, C. S. O’Hern, and M. Dennin, Phys. Rev. E 77, 041505 (2008).

\section{ii. Step strain measurements}

Many of the interesting behaviors in complex fluids occur at extremely slow rates of strain. Often, these conditions are modeled using quasistatic simulations in which small steps in strain are made and then the system is equilibrated. The assumption is that the behavior in the limit of continuous rate of strain going to zero is the same as the behavior under quasistatic strain. However, important differences between simulations and experiments exist, and it is not clear if these are due to difference between continuous and quasistatic strain, or to other effects. Therefore, we carried out a systematic comparison of step strains and continuous strains.

The main result of these initial studies was published in Comparison between step strains and slow steady shear in a bubble raft, M. Twardos and M. Dennin, Phys. Rev. E 71, 061402 (2005). Essentially, the focus was measurements of the distribution of stress drops during "flow". This is a common method for characterizing the behavior of slow flows. As the foam, or bubble raft, is strained, the stress (or energy) increases and decreases in an irregular manner. The periods of decrease are referred to as stress drops. The statistics of these stress drops are different in the various models, and even in some experiments. One measure of the distribution of stress drops is the average size of a stress drop. In simulations based on quasi-static steps, the average stress drop is found to increase and then plateau as the effective strain rate is reduced. (Here the effective strain rate is the amount of strain during the step divided by the waiting time for each step.) In contrast, our work with continuous strain found a decrease in the average stress drop and then a plateau. By directly comparing with step-strains, we determined two things. (1) The experimental step-strain behavior is consistent with simulations and differs from the continuous behavior using a standard definition of a stress drop. (2) Using an alternative definition of a stress drop that emphasizes a typical time for an "event", the behavior during continuous strain and step strains agreed with each other. Putting the results together provides strong evidence that events have a "typical time", and that multiple time scales play a role in determining the behavior of slowly sheared foam.

As an important next step, we will study the details of the behavior during a step strain using the parallel trough. This will allow us to look at distribution of events after a step strain in a geometry with uniform stress.

\section{iii. Boundary condition}

One of the main results of the project was the observation of a unique type of flow localization: the coexistence of a flowing and a jammed state. This result appeared to 
contradict previous work by another group that suggests single layers of bubbles in a Couette flow localize with an exponential profile near the inner boundary. Two differences between the experiments were the bottom support for the bubbles and the top boundary condition. In our Couette apparatus, the bubbles are supported by a fluid subphase and the top is open to the air. In contrast, the other experiment was for bubbles confined between two glass plates.

To better understand the differences between the experiments, we carried out measurements on the flow behavior for a layer of bubbles with a free top and one in which the top is in contact with a glass plate. We found that the top dramatically altered both the velocity profile and the distribution of bubble rearrangements. Without a top, flow and bubble rearrangements are observed throughout the system. With a top, motion is confined to the first row or two of bubbles. This is true even for very slow flows for which groups have previously made the argument (without experimental verification) that the interaction with a top or bottom boundary is not relevant. Our experiments demonstrate that even at slow speeds the interactions with a confining plate can not be ignored. This work appeared in Impact of boundaries on velocity profiles in bubble rafts, Y. Wang, K. Krishan, and M. Dennin, Phys. Rev. E 73, 031401 (2006).

\section{iv. Analysis software and webpage development}

Another major area of focus has been the development of a complete analysis software package for the bubble raft experiments. This package was developed in Matlab, and is intended for general use with two-dimensional bubble systems. We have made it available on our website for use by other groups.

\section{Summary of other results}

We have looked closely at the fluctuations in the velocity. This quantity is useful for making connections to proposed theories concerning effective temperatures. Also, it is a quantity that has been studied within the context of various models of foam, so it provides a useful test of theoretical models. We found general agreement with most features of the models. However, we observed an interesting non-Gaussian tail to the velocity fluctuations that had not been reported for simulations. This is useful as it provides information regarding the limitations of existing models. This work is being continued in the parallel trough, where the experiments are easier to interpret because the velocity profiles are linear. We have access to a wider range of rates of strain, and better statistics at each rate of strain value. The better statistics will allow for a more careful characterization of the non-Gaussian tails of the distribution.

Our work on fluctuations in the plastic bead rafts has used the information gained concerning fluctuations in stress (published in Slow Steady Shear of Plastic Bead Rafts, Michael Twardos and Michael Dennin, Granular Matter 7, 92 (2005). ) and moved to studies of fluctuation-dissipation relations. The fluctuation-dissipation relations that are of most interest in these systems are based on comparing the correlation function for a 
fluctuating quantity (for example, the strain) to the related response function (for example, the response function for an applied stress). The study of generalized fluctuation-dissipation relations has become very important in characterizing the flow behavior of a wide range of complex fluids. Two aspects are of interest: (1) violation of standard fluctuation-dissipation relations and (2) applications of fluctuation-dissipation relations for defining effective temperatures. Our measurements of fluctuation-dissipation relations for the plastic beads have revealed a very interesting dependence of the response function on the direction of the applied stress, even for a regime in which the stress should be small enough that linear response applies. This asymmetry is very indicative of the existence of stress chains. These studies were published in Asymmetric response of a jammed plastic bead raft Michael Twardos, Michael Dennin, Phys. Rev. Lett. 97, 110601 (2006). Additional work on fluctuations and their impact on determining average values was published in Limits of the Equivalence of Time and Ensemble Averages in Shear Flows, Y. Wang, K. Krishan, and M. Dennin, Phys. Rev. Lett. 98, 220602 (2007).

Finally, we have made progress in the small side project that was motivated by our general exploration of "jamming": voltage quenches in electroconvection. This is a system that was previously studied in our lab for the purposes of exploring completely different questions. However, recent theoretical work predicted that a glassy transition should occur in this system due to defect motions. We felt this connected to the work on bubbles in the context of the general issue of how defects (or other topological features, such as T1 events) control the transition from a mobile state to a jammed state. In electroconvection, a pattern of convection rolls is formed in a nematic liquid crystal. The pattern has topological defects that move in a periodic background (the pattern). Our initial results have indicated that a jamming transition might be relevant in this system. We have determined that this work is an ideal training ground for undergraduate students in many of the techniques that are used to understand the bubble experiments. Therefore, we continue to use an existing apparatus to provide such training, and these students generally have the option to switch to an appropriate bubble project once they have sufficient mastery of the necessary skills.

III. Student Support and Additional Activities.

The grant supported two graduate students:

Mary Griffith for February and March received MA June 2005 (completed her Masters under a fellowship from the Department of Physics and Astronomy at UCI, and is currently employed at Raytheon in the Los Angeles, CA area).

Michael Twardos for February and March received Ph. D. March 2005 (currently a postdoctoral researcher at Los Alamos National Laboratories).

The grant is supported one postdoctoral researcher who is taking a major role in the project:

Kapil Krishan May-October (2005) 
There has also been some support for an undergraduate researcher:

Scott Sullivan April, May, June, September, October (2005)

Finally, two additional graduate students have been working on the project, but they are currently supported as teaching assistants in order to fulfill their teaching requirements.

\section{Yuhong Wang} Myles Adams

Because of the work funded by this grant, I have initiated the development of a course on Continuum Mechanics at the graduate level that provides students the necessary background to work in this field. As the course evolves, the plan is to develop a textbook based on the course. Also, because of this work, I have been invited to give a number of presentations on integrating research in this area into the undergraduate curriculum. Finally, this has proved a fruitful area for outreach at the K-12 level. As such, I will be giving a workshop on introducing K-12 students to the science of foam and sand.

\section{Current Federal Support:}

Current Support $\quad \$ 309,000 \quad 7 / 01 / 2009-6 / 30 / 2012$ NSF-DMR Biomaterials "Collaborative research: Mechanics and Microrheology of Biomemtic Materials" University of California at Irvine Months committed per year: 1 